\title{
NOTA SOBRE UNA ATRIBUCIÓN AL MANCEBO DE ARÉVALO
}

\author{
Luis F. Bernabé Pons*
}

La profesora María Teresa Narváez, en su edición de la Tafsīra del Mancebo de Arévalo, a la hora de hablar de otras posibles obras que pudiera haber escrito el autor castellano indica en nota al pie de página que "parecería que el ms. J12 contiene unos capítulos que también se hallan en el 'breve conpeño, que fue sacado de un mançebo y de un alfaquí con él' [...] mientras el ms. J 52 incluye una disertación ofrecida en Ávila la Real por una mora a unos sabios [...] dato que recuerda poderosamente a un episodio mencionado por el Mancebo y relativo a una exposición que hace Nozaița Kalderán"1 . La mención que se hace al Breve compendio de nuestra santa ley y sunna en el famoso párrafo del manuscrito aljamiado J12 del C.S.I.C. ya ha sido traído a colación en diversas ocasiones para mostrar aspectos de la fama del Mancebo de Arévalo $^{2}$ entre los moriscos aragoneses:

Y dexta manera lo hallamox también en el libro xogubiano por xu autor del libro. Y anxí mesmo lo hallamox en otro libro en la Ribera que se llama el brebe conpeño, que fue xacado de un mancebo y de un alfaquí con él, y fue corregido de lox sabios de Aragón y de lox alfaquíex por cuanto el autor del libro era castellano y de gran çençia en el adín del alislam ${ }^{3}$.

Ese "lo hallamos" se refiere a la materia islámica que acaba de desarrollar el manuscrito J12, una serie de ordenaciones y consejos acerca de la oración, la limosna y el ayuno. Parecería que el fragmento se refiere, de una manera genérica, a que esa materia, o el desarrollo de ella, se halla asimismo en el compendio legal de 'Isā ibn Ŷābir, el Breviario sunní (= libro segoviano) y en el Breve

\footnotetext{
* Universidad de Alicante.

1. Tratado [Tafsira]. Mancebo de Arévalo, Edición, introducción y notas de María Teresa Narváez Córdova, Madrid, 2003, p. 15, n. 5.

2. Véase, por ejemplo, M.J. RUBIERA, «El Islam cristianizado de los moriscos castellanos en época de Carlos V», en Carlos V y la quiebra del humanismo político en Europa (1530-1558), Madrid, 2001, pp. 469-485.

3. J. Ribera y M. Asín, Manuscritos árabes y aljamiados de la Biblioteca de la Junta, Madrid, 1912, p. 71. En esta cita y las siguientes que se hagan, respetamos las transcripciones hechas por cada autor. Si no se cita ningún autor, se sobreentiende que la transcripción es mía.
} 
compendio que, como es conocido, se presenta como el trabajo en colaboración del Mancebo de Arévalo y el alfaquí Ibrāhīm de Remincho ${ }^{4}$. Nada nuevo, pues, en lo que conocemos de la importancia de la obra del muftí de Segovia en el mundo morisco aragonés y en el papel del Mancebo en la difusión de su obra ${ }^{5}$.

La segunda de las referencias relacionadas con el Mancebo de Arévalo, sin embargo, parece ofrecer algo más concreto en tanto que se menciona una disertación ofrecida por una mora. Podemos atender a esas breves palabras que una innominada mora dedica a unos musulmanes en el manuscrito J 52:

\section{[Basmala]}

Éste es un capítulo todo de los dichos de los sabios sacado de arábiga (sic) en romançe para dexenplo a los muslimes, que los dixo el annabí Muhammad, ș $\mathrm{s}^{\mathrm{c}} \mathrm{m}$.

Fue recontado que estando en Ávila la Real una compaña de sabios onrados tratando de la sabiduría en el adín del alislam, en las cosas de los așalaes del deudo y de los așalaes de anefilas y de todas las demás cosas del deudo y de las cosas de graçia en el hazer dellas, en este estante dentró una mora onrada muy dota en el saber del adín del alislam y dixo: “Donde ay tanta nobleza de sabios yo no puedo hablar, mas con su licencia yo diré mi pareçer en esa convesaçión (sic)". Y dixéronle que en aquella y en todo lo demás tenía licencia para poder hablar en las cosas del addín del alislam. Y dixo la mora onrada: "Las anefilas son buenas y de mucho (muŷo) valor en poder de Allah, y las aṣadaqas de graçia son buenas y de mucho valor en poder de Allah, pero lo mayor y más aventajado y más amado en poder de Allah son los deudos aquellos que aduedeçió Allah en su onrado Alqur'en sobre sus siervos". Y dixeron todos: "Ella a dicho lo último de la verdad"6.

Éste es el fragmento que en mi opinión puede atribuirse claramente a esa mora que interviene en la reunión con el permiso de los allí presentes, puesto que tras estas líneas el texto parece tomar otros derroteros. Como señala la profesora Narváez, el que una mora intervenga ante un auditorio en Ávila la Real, recuerda mucho a un episodio protagonizado por Nozayța, Nuzaya o Nuzayla Kalderán en el Breve compendio de nuestra santa ley y sunna, contenido en el Manuscrito Dd. 9.49 de la Biblioteca Universitaria de Cambridge, y dado a conocer en diversos trabajos por el profesor Leonard P. Harvey, quien prepara asimismo la transcripción completa del códice. No es, por supuesto, Nuzayța

4. L.P. HarveY, «El alfaquí de Cadrete, Baray de Reminjo y El Breve Compendio de Nuestra Santa Ley y Sunna», II Jornadas Internacionales de Cultura Islámica. Aragón vive su historia (Teruel: 1988), Madrid, 1990, pp. 213-222; L.F. BERnABÉ PONS, «Nueva hipótesis sobre Baray de Reminŷo», Sharq Al-Andalus, 12 (1995), pp. 299-314.

5. L.P. HARVEY, «El mancebo de Arévalo y la tradición cultural de los moriscos», en Á. GALMÉS DE FuEntes (dir.), Actas del Coloquio Internacional de Literatura Aljamiada y Morisca (Oviedo: 1972), Madrid, 1978, pp. 20-41; G. WIEGERS, Islamic Literature in Spanish and Aljamiado: Iça of Segovia (fl. 1450). His antecedents and Successors, Leiden, 1994.

6. Ms. J 52 del C.S.I.C., fol. 256 r. 
Kalderán ninguna desconocida para todo aquél lector interesado en el Mancebo de Arévalo7: anciana extranjera que ha recorrido numerosos países islámicos antes de llegar a España, recorre los caminos, especialmente de Castilla, ejerciendo de partera y maga o curandera. Parece ser, al mismo tiempo, figura respetada y reclamada entre los moriscos, que la conocen a causa de sus conocimientos islámicos, y el mismo Mancebo de Arévalo tiene ocasión de disfrutar de sus enseñanzas e incluso de tener algún encontronazo con ella ${ }^{8}$.

El episodio concreto recogido en el Breve compendio se halla narrado a partir del folio $34 \mathrm{v}$ y se encuentra entre las partes transcritas por Harvey en su tesis doctoral. En él la discusión entre los musulmanes gira en torno al mismo tema de si las oraciones voluntarias (șalāwāt an-nāfila o șalāwāt as-sunna) tienen el mismo valor que las oraciones obligatorias (șalāwāt al-fard o șalāwāt aljamsa), pero la conversación se plantea con algo más de detalle?:

I porke biyene al kašo diremoš lo ke šobre ešte kašo šusediyo en Abila la Rreal un diya de loš šiyete del año. Entre muy onrradoš muslimeš še lebanto una porfiya maš pešada ke lo konfiyaba de peršonaš tan heziyentes komo alli se hallaron. Unoš deziyan ke todo hacedor de așṣala biyen compuesto i kon buena aniya teniya kompañiya de almalakeš. Deziyan otroš ke loš que haziyan așsala de presebto fuwera de la ora no teniyan almalakeš, y ke akel așșala no še alu aba kon așșala hecho en sin ora, šino de parte, porke šu kantidad no era tal...

Tornaron a rrepilikar los pirimeroš ke puweš todo er(a) așșala, no deššariya de šer en apalazo de su dibina bondad, i ke no šeriya šeparante el un așșala del oro, aunke no fuweše tan noble el uno komo el otro, y ke para šu bondad, no era menester taratar adefešiyoš, porke todo le era perešente.

Deziya la otra parte ke todo akello era verdad, mas ke la šeparansa de loš aṣșalaes la aporobariyan kon teštoš carabigoš. Y al fin de muchas alterkansaš, determinaron imbiyar a llamar a Nuzaya Kalderan, ke a la šazon eštaba alli en Abila, kurando un parto dešfallesido. I kuwando bino al cabildo de la porfiya, todoš le hiziyeron mucha onor, i diššeronle toda la kawša šin pašiyon, y rrešpondiyo enta todoš kon una severidad ke nunca yo bi maš ešenta, i diššo anši:

“Yo temo ke por kualkiyere biya tengo de šer la denoštada, pero al fin digo ke todaš laš diziyendaš de nuwestoro onrrado alquren šon perekalaraš ši laš šabemoš entender, porke dize en šu diziyenda no a de aber maš šubisiyon de la ke

7. Véase especialmente M.T. NARVÁEZ CÓRDOVA, «Nozaița Kalderán: partera y experta en el Corán», La Torre, III-IV (1987), pp. 501-517 [También en A. TemiMI (ed.), Actas del III Simposio Internacional de Estudios Moriscos. Las Prácticas Musulmanas de los Moriscos Andaluces (14921609), Zaghouan, 1989, pp. 139-150]. Una reciente hipótesis sobre su identidad en M.J. RUBIERA MATA, «Las dos orillas cervantinas. A modo de introducción con addenda», en M.J. Rubiera Mata (ed.), Cervantes entre las dos orillas, Alicante, 2006, pp. 9-20 (p. 17).

8. Tafsira, pp. 61-64.

9. The literary culture of the moriscos (1492-1609): a study based on the extant mss. in Arabic and Aljamia, Oxford, 1958, vol. II, pp. 80-83. Tesis doctoral inédita que manejo con el generoso permiso del Prof. Harvey. 
še noš puwede manifeštar literalmente, porke eš nuweštoro onrrado alquren de tal kondisiyon ke tan fácilmente še denoštara a un na uwe komo a un mediano tarašsender, kada uno en šu kištiyon.

A kuwanto el akompañamiyento de loš almalakeš, yo digo ke todoš kedayš libreš šegin (sic) mi razón, i lo ke mi entendimiento denota. No puwede deššar de tener kompañiya de almalakeš todo fazedor de așșala, en la ora o fuwera de la ora, por esta biya ke dire: el ke haze așșala en šu ora, komo lo manda nuwestoro onrrado alquren, ešte tiene loš almalakeš muy apersebidaš y šubelanteš, i por ešto rrešerbaron loš almalakeš al omisiyente ke abiya hecho treynta omisidas i šuš pekadoš. Keriyanšelo lebar a `ahannam, i loš almalakeš šubiniyenteš defendieron el arršǔš, porke lo alkanso šu palazo en la ora del așșala, porke bean los muslimeš de kuwanto pešo eš la ora ašignada del așạala. También še puwede hazer așsala en la ora i šer tan deškonpuwešto ke sešen loš almalakeš de akella pelenitud ešensiyatiba ke ušan loš fazedoreš puntuwales.

Loš ke hazen așșala fuwera de la ora, no loš acompañan loš almalakeš kon akella perekalaransa del punto decretado, keš la ora del așșala, i ši a eštoš loš topaše la muwerte komo topo al dicho omisido, eš Allah šabidor de tal fecho".

El discurso continúa durante un par de páginas más, insistiendo en que así lo señala el Corán y mejor es no osar interpretar el texto sagrado. La oración mejor es el "așșala de la puntualidad, ke noš kita todaš laš dudaš", y las horas de la oración fueron establecidas por Dios como "llabeš de laš adduwanaš del siyelo". Cabe interpretarse, pues, que según la sabia Calderán, todas las oraciones que hace el musulmán son buenas por naturaleza y comportan beneficios para el creyente, pero las oraciones obligatorias prescritas en el Corán son las que mayor virtud y amor de Dios recaban para el creyente y la comuni$\mathrm{dad}^{10}$, por encima de las oraciones voluntarias, no sujetas a pauta horaria.

Una comparación de ambos textos parece llevar fácilmente a la conclusión de que el fragmento recogido en el manuscrito J 52 y los parágrafos del Breve compendio se refieren a la misma escena: la de una mora sabia que está resolviendo dudas acerca del auténtico valor de las oraciones no obligatorias. Sin embargo, el primer manuscrito recoge la escena y el discurso de forma muchísimo más breve que el segundo, sin mencionar el nombre de Nuzaya Kalderán ni su actividad y, por supuesto, recortando muchísimo sus palabras. De hecho, en el manuscrito J 52 el discurso pierde toda la controversia de opiniones e igualmente las consideraciones sobre los ángeles que están presentes en las oraciones de los musulmanes, limitándose simplemente a una escueta conclusión.

10. M.J. CERVERA FrAS, «Jaculatorias para toda la jornada en el manuscrito 505 de Toledo», en A. TEMIMI (ed.), Actes du IV Symposium International d'Etudes Morisques sur: Métiers, vie religieuse et problématiques d'histoire morisque, Zaghouan, 1990, pp. 100-110; ID., «Notas sobre la rogativa en el islam mudéjar», en Homenaje a la profesora Carmen Orcástegui Gros. Aragón en la Edad Media, Zaragoza, 1999, XIV-XV, vol. I, pp. 291-301; N. MARTíneZ DE CASTILLA, «Cohesión y control: la oración de los moriscos a través del ms. T19 (R.A.H.). Documentos escogidos», IX Simposio Internacional de Mudejarismo. Actas, Teruel, 2004, pp. 165-179. 
Parece que el manuscrito J 52 en este punto efectivamente está resumiendo lo que existía en un manuscrito anterior y era conocido por los moriscos más cultos. Teniendo en cuenta que este manuscrito puede datarse aproximadamente en $1599^{11}$ y que las obras del Mancebo son por fuerza anteriores a esa fecha, no parece extraña esa relación. No hay que olvidar que la fama del Mancebo de Arévalo entre la comunidad morisca aragonesa debió de ser notable, incluso después de su muerte. Ya se ha citado el trozo del manuscrito J 12 que habla de la "ciencia" del escritor castellano, pero puede recordarse asimismo cuánto debe al Mancebo la obra poética del morisco de Rueda de Jalón Muhámad Rabadán ${ }^{12}$, ya en el alba del siglo XVII.

Estaríamos, pues, no ante un fragmento original de Mancebo de Arévalo, sino ante un fragmento de una mano posterior que, basándose en el relato del Mancebo (leído o escuchado), reproduce esquemáticamente la escena. Su inclusión dentro de este manuscrito que contiene obras de diversa índole remitiría a un criterio de autoridad que parecen haber cobrado las obras del escritor musulmán castellano.

El manuscrito J 52, inédito hasta donde se me alcanza, con excepción de unos poemas en alabanza de Dios y del islam editados y estudiados por Toribio Fuente Cornejo ${ }^{13}$, no parece contener ningún otro fragmento relacionado con las obras del Mancebo de Arévalo. Sin embargo, en la misma parte en la que se halla la referencia a la alocución de la mora en Ávila, pueden leerse también algunos interesantes fragmentos que le hacen merecedor de algún estudio más detenido. Así, por ejemplo, el fragmento que se encuentra entre los folios 265 r y 266 r trata el tema de los musulmanes que viven en tierra de infieles. El profeta Muhámmad explica los grandes méritos de estos creyentes y las altas recompensas que les esperan. El tema es antiguo y conocido, y aparece en otros manuscritos moriscos ${ }^{14}$, pero el manuscrito J 52 introduce un elemento novedoso al incluir tras las palabras del profeta del islam una consideración, a todas luces contemporánea del morisco, acerca de la dificultad de pasar a tierra de musulmanes y de los méritos que tendrá aquel buen musulmán que permanezca en tierra infiel. Incluso al final del texto, el propio escribano se permite dar en primera persona -cosa bastante inusual- su opinión sobre lo que él mismo está escribiendo, lo que da un inesperado golpe de realismo al texto:

11. J. Ribera y M. Asín, op. cit., p. 192.

12. J.A. Lasarte López, Poemas de Mohamad Rabadán. Canto de las lunas - Día del Juicio - Discurso de la Luz - Los nombres de Dios, Zaragoza, 1991.

13. Poesía religiosa aljamiado-morisca (Poemas en alabanza de Mahoma, de Alá y de la religión islámica. Otros textos complementarios), Madrid, 2000, pp. 265-274.

14. Véase L.F. BERNABÉ PONS, «Laus al-Andalus en la literatura morisca», Revista del Instituto Egipcio de Estudios Islámicos, XXVI (1993-1994), pp. 149-160. 
(f. 265 r) Fue recontado por el annabí Muhammad, ș $\mathrm{c}$, que él dixo estando un día con los de la șihaba, dixo:" Abrá una puerta de los de mi alumna en una isla que se llamará la isla de l-andaluzía, que aquéllos serán los más fuertes guerreros en fī sabili Lāhi de todos los del adīn del alislām, y ternán tanto gualard(ón) para ello en poder de Allāh como setenta de vosotros". $Y$ dixeron: " ¿Como setenta dellos, yā Rasūl Allāh?". Y dixo el annabí Muhammad, ș̣ m,: “No, sino como setenta de vosotros". Dixéronle: “¿Pue(s) y cómo será aque(l)lo, yā Mensajero de Allāh?”. Díxoles, șcm: “¿Porque ellos guerrearán cuentra los descreyentes sin armas de noche y de día y se defenderán dellos con alhelas y buenas palabras, y sus corazones estarán firmes en la palab(r)a de lā ilāhi illā Allāh, Muhammad Rasūl Allāh, como la bola emplomada ${ }^{15}$ fuerte en los corazones. Y el día del Juicio los levará Allāh a la-l-ŷanna por su piadad a ellos y a sus barras ${ }^{16}$.

Fue recontado por uno de los grandes sabios que castigando un día a las gentes que se fuesen desta tierra a la tierra de los(s) muslimes a vivir y que allí estarían más en el se(r)viçio de Allāh y en su obidençia. Díxole uno de los que estaban con él de los desta tierra: “¿Cómo abemos de pasar en tierra de muslimes? Que si imos enta delante, está la mar; y si imos enta tras, está el fuego de la inquisición; y si imos enta la mano derecha están los lad(r)ones para robarnos, matarnos; y si imos enta la mano izquierda están los enemigos para prendernos. Y nosotros estamos derrenquili- (f. 265 v.) -dos al juzgo de Allāh y a su piadad y a deguardar aquello que Él será sabido de hazer sobre nosotros en este mundo y en el otro". Dixo el sabio: "Yo digo que en todo lo que dezís conozco que tenéis razón y que dezís verdad, y que Allāh es el hacedor de lo que quiere en los çielos y en la tierra, y Él es sobre toda cosa poderoso y juzga sobre sus ja-

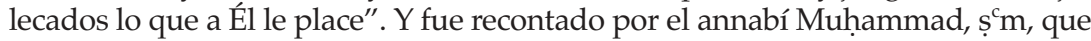
él dixo también: "Aventurado será aquél que vivrá en tierra que son malos y él será bueno". Y digo yo, el escribidor destas palabras: "También aventurado será el que viva entre los descreyentes con Allāh, y él será siempre con Allāh, y averdadeçerá con la mensajería de Muhammad, $\mathbf{s}^{\mathrm{c}} \mathrm{m}$, (266 r) y especialmente si cumple con los deudos y mandamientos de Allāh, el Señor de todas las cosas".

\section{RESUMEN}

Análisis de un fragmento del manuscrito aljamiado J 52 del Consejo Superior de Investigaciones Científicas del que se ha sugerido que podría pertenecer al Mancebo de Arévalo. Comparación de dicho fragmento con otro similar de una obra del Mancebo.

Palabras clave: aljamiado, Mancebo de Arévalo, moriscos de Aragón.

15. En el ms. Enpalomada.

16. En el ms. Perraš o Pārraš. Interpreto así, a partir de la raíz b r r, más el sufijo plural castellano, como es usual en los textos aljamiados, como "buenas obras". 


\begin{abstract}
Analysis of a fragment of the aljamiado manuscript J 52 of the Consejo Superior de Investigaciones Científicas (Madrid) that supposedly might have been writed by the Mancebo of Arévalo. Comparison of this fragment with another similar one of a work of the Mancebo.
\end{abstract}

Key words: aljamiado, Mancebo de Arévalo, moriscos from Aragon. 